\title{
Garcinia binucao crude ethanolic leaf extract prevents alcohol- induced neurotoxic effects on learning, short-term memory, and motor functions in Drosophila melanogaster
}

\author{
Ourlad Alzeus G. Tantengco ${ }^{1}$, Joanne Jennifer E. Tan ${ }^{1}$, Nicholas Robert C. Tan ${ }^{1}$, Maria Concepcion C. Sison², Paul Mark B. Medina ${ }^{1 *}$ \\ ${ }^{1}$ Biological Models Laboratory, Department of Biochemistry and Molecular Biology, College of Medicine, University of the Philippines Manila, Pedro Gil \\ St., Ermita, Manila, Philippines \\ ${ }^{2}$ Department of Pharmacology and Toxicology, College of Medicine, University of the Philippines Manila, Pedro Gil St., Ermita, Manila, Philippines
}

\begin{tabular}{|c|c|}
\hline ARTICLE INFO & ABSTRACT \\
\hline $\begin{array}{l}\text { Article history: } \\
\text { Received on: } 17 / 03 / 2018 \\
\text { Accepted on: } 25 / 08 / 2018 \\
\text { Available online: } 31 / 10 / 2018\end{array}$ & $\begin{array}{l}\text { Excessive alcohol consumption is associated with cognitive and behavioral impairments. Drosophila melanogaster } \\
\text { is a commonly used model for learning, memory, and motor function studies. We looked at Garcinia binucao to } \\
\text { determine the ability of its crude ethanolic leaf extract to reduce deficits in learning, short-term memory, and motor } \\
\text { functions due to alcohol exposure. Chronic alcohol exposure significantly decreased motor function by } 22 \%(\mathrm{p}< \\
0.05) \text {, learning function by } 37 \%(\mathrm{p}<0.05) \text {, and short-term memory by } 33 \%(\mathrm{p}>0.05) \text {. However, in flies fed with }\end{array}$ \\
\hline $\begin{array}{l}\text { Key words: } \\
\text { Garcinia binucao, } \\
\text { Drosophila melanogaster, } \\
\text { alcohol-induced } \\
\text { neurotoxicity, learning, } \\
\text { short-term memory, motor } \\
\text { function. }\end{array}$ & $\begin{array}{l}\text { G. binucao extract (GBE) chronically exposed to alcohol effects of certain compounds, no significant reductions in } \\
\text { motor, learning and in short-term memory functions were observed; instead, these functions increased }(\mathrm{p}<0.05) \text { in } \\
\text { a dose-dependent manner, suggesting that the extract is not only neuroprotective but is also potentially nootropic. } \\
\text { GBE had low antioxidant activity despite the presence of triterpenes, flavonoids, and quinones, although these } \\
\text { may have contributed minimally to the neuroprotective ability of GBE. The neuroprotective ability of GBE may } \\
\text { then be explained by other mechanisms not dependent on the direct radical or oxidant scavenging properties of the } \\
\text { phytochemicals in GBE, as in the modulation of signaling cascades which prevent neuronal apoptosis. }\end{array}$ \\
\hline
\end{tabular}

\section{INTRODUCTION}

Alcohol consumption is known to be highly prevalent in the general adult population. According to WHO 2010 data, $47.7 \%$ of males and $28.9 \%$ of females worldwide are current drinkers (WHO, 2014). Approximately $38.9 \%$ of Filipinos are occasional alcohol drinkers, $11.1 \%$ are regular drinkers, while $4.8 \%$ are heavy drinkers (Vinluan, 2003). It has been shown that the frontal lobes, limbic system, and cerebellum are vulnerable to damage caused by alcohol in a dose-dependent manner (OscarBerman and Marinkovi, 2014).

Several researches on natural products have been

"Corresponding Author

Paul Mark B. Medina, Biological Models Laboratory, Department of Biochemistry and Molecular Biology, College of Medicine, University of the Philippines Manila, Pedro Gil St., Ermita, Manila, Philippines. E-mail:pmbmedina@post.upm.edu.ph conducted to discover new drugs which may improve the quality of life, serve as therapy for the disease, or neutralize the toxic effects of certain compounds including alcohol. The Philippines is known to have a diverse source of medicinal plants. Garcinia binucaowhich belongs to the family Clusiaceae, is endemic and widely distributed in the Philippines, particularly in the Visayas region, including Negros Occidental, Iloilo, Samar, Leyte (Quevedo et al., 2015). Aside from being a source of food, G. binucao is also noted for its folkloric medicinal properties. In the Cordillera region, G. binucao is used for suppressing "agas ti nginao" (conceiving mothers' cravings), cough, flu, and arthritis (Chua-Barcelo, 2015). Its fruit and leaves are also used as a souring agent in different provinces in the Philippines. Despite its prevalence and widespread use, there have been few studies on G. binucao. However, a close relative, Garcinia mangostana, more commonly known as mangosteen, has been hailed as a "super fruit" health supplement with anti-cancer, anti-inflammatory, and anti-neurotoxic activities 
(Pedraza-Chaverri et al., 2009; Gutierrez-Orozco and Failla, 2013; Tangpong et al., 2012). Phytochemical analysis of various extracts from several Garcinia species also yielded several antioxidant compounds, such as xanthones, triterpenoids, carotenoids, phenolic compounds and biflavonoids, all of which are established components in plant anti-oxidant systems (Rukachaisirikul et al., 2000, Hemshekhar et al., 2011). G. binucao, thus, has high potential to also possess these compounds and the accompanying properties.

Considering prevalent chronic alcohol consumption in the population which may lead to ethanol-induced neurotoxicity, and the known widespread use of $G$. binucao which may have potential anti-neurotoxic properties, this study aims to determine the effects of $G$. binucao crude leaf extract on the motor, learning, and short-term memory functions of $D$. melanogaster against the neurotoxic effects of chronic ethanol exposure, as well as sexspecific differences that may be observed.

\section{MATERIALS AND METHODS}

\section{Specimen collection and crude extraction}

One kilogram of $G$. binucao leaves were collected from Sitio Pastolan, Tipo, Hermosa, Bataan. A voucher specimen was prepared and submitted to the Botany Division of the National Museum of the Philippines for taxonomic identification.

The collected $G$. binucao leaves were washed thoroughly with distilled water and air dried at room temperature. Dried samples were then crushed using a blender until uniformly powdered. Fifty grams of the powdered samples were soaked in $500 \mathrm{~mL}$ of $95 \%$ ethanol for 72 hours and filtered using Whatman filter paper grade no. 41 . The filtrate was evaporated under reduced pressure through rotary evaporation.

\section{DPPH assay}

Sample solutions, $400 \mu \mathrm{g} / \mathrm{mL}$ of the crude leaf extract and ascorbic acid as positive control were prepared and tested for antioxidant activity (Prieto et al., 1999). In a 96-well plate, $200 \mu \mathrm{L}$ of the sample (in triplicate) was loaded in the first well and a twofold serial dilution was performed until the $12^{\text {th }}$ well using buffer solution. Then $100 \mu \mathrm{L}$ of $0.2 \mu \mathrm{M}$ DPPH solution was added per well. The 96-well plate was incubated in the dark for 30 minutes at room temperature. Absorbance at $517 \mathrm{~nm}$ was read. Percent scavenging activity and EC50 values were computed.

\section{Phytochemical analysis}

Phytochemical analysis was performed in the Natural Products Laboratory of the Department of Biochemistry and Molecular Biology, the University of the Philippines Manila using 1 gram of the crude leaf extract.

\section{Drosophila culture and husbandry}

Culture media preparation and longevity assays used in the study were based on the previous method (Velasco and Medina, 2014). The mass culture of Drosophila melanogaster used in this study was maintained on sweet potato-yeast media consisting of $500 \mathrm{~g}$ of sweet potato, $10 \mathrm{~g}$ of yeast and $15.4 \mathrm{~g}$ of agar in $1000 \mathrm{~mL} \mathrm{dH_{2 }} \mathrm{O}$. The entire mixture was allowed to boil and to cool down to $60^{\circ} \mathrm{C}$ before adding 8 drops of pure propionic acid in order to inhibit the growth of microorganisms. The food media were stored at constant temperature and humidity in the laboratory. The same formulation for the food media was used for growing several generations of D. melanogaster.

For all subsequent assays, fruit flies were separated based on sex. The separation of sexes is important for the assays because the flies should not be allowed to mate to prevent an increase in the initial population. The flies were anesthetized first using the $\mathrm{CO}_{2}$. A few shots of $\mathrm{CO}_{2}$ were pumped into the vial through the cotton plug. The anesthetized fruit flies were then transferred to the fly pad. Sorting and sexing was done with a stereomicroscope by observation of sexual dimorphism (e.g., males are generally smaller and have a darker, more rounded abdomen, and tarsal sex combs on their first pair of legs).

Separately, 30 males and 30 female flies were used in each assay. Four treatments were used in all the assays. For the control set-up, the food media were used without addition of neurotoxic solvent, dimethyl sulfoxide, and G. binucao leaf extracts. It had the same formulation as to the food media used for growing generations of $D$. melanogaster.

\section{Alcohol-induced oxidative stress and neurotoxicity}

The vial plug was flooded with $0.5 \mathrm{~mL}$ of absolute alcohol and inserted in such a way that the ethanol-flooded side of the plug faced the flies. Flies were exposed to ethanol for five minutes daily. After each ethanol exposure, the flies were returned to their food vial. This procedure was done for 15 days after which the behavioral assays were then performed.

\section{Negative geotaxis assay}

Fruit flies that have been subjected to chronic alcohol exposure were then subjected to the negative geotaxis assay (Berger et al., 2011). Motor deficits resulting from ethanol-exposure toxicity were measured using the negative geotaxis assay. Eight groups of thirty flies: food-only control, ethanol-exposed control, 1\% DMSO, ethanol-exposed 1\% DMSO, $1 \mathrm{mg} / \mathrm{mL} \mathrm{G.} \mathrm{binucao,}$ ethanol-exposed $1 \mathrm{mg} / \mathrm{mL}$ G. binucao, $200 \mu \mathrm{g} / \mathrm{mL}$ G. binucao, and ethanol-exposed $200 \mu \mathrm{g} / \mathrm{mL}$ G. binucao were placed in three different graduated vertical columns sealed at one end. The flies were allowed at least an hour to recover from the $\mathrm{CO}_{2}$ anesthesia and a minute to acclimatize to the new setting. The glass columns were then gently tapped to let the flies settle at the bottom of the vial. Subsequently, climbing activity was observed for a minute. The number of flies per group passing the $8 \mathrm{~cm}$ minimum height marker for every 10 -second interval for one minute was measured and noted as a percentage of the total flies without observed motor deficits. Conversely, flies with motor deficits remained near the bottom or were slow-moving. This procedure was done for a total of three times per replicate.

\section{Aversive phototaxis suppression assay-learning assay}

Fruit flies that have been subjected to chronic ethanol exposure were then subjected to the aversive phototaxis suppression assay (Berger et al., 2011). A fly was then placed inside the dark chamber of the T Maze. All lights in the room were turned off with the exception of the red lamp. The fly was allowed to acclimatize to the dark chamber for around 30 seconds after which the light source from the lighted chamber was turned on 
slowly. The trap door was then opened gradually to connect the two chambers. The fly was determined as positively phototactic and ready for training if it moved to the lighted chamber within 45 seconds. Otherwise, the fly was noted as negatively phototactic and was excluded from the experiment.

The positively phototactic fly was relocated back to the dark chamber. The trap door and light were then closed and the fly was allowed to acclimatize to the dark chamber once more for 30 seconds. Filter paper treated with quinine solution was then placed into the lighted chamber. Once the fly was acclimatized, the trap door and light were then slowly opened. The fly was allowed to walk into the lighted chamber with quinine treated filter paper. After 45 seconds, the fly was tapped back to the dark chamber, and the process was repeated 14 more times. Flies that failed to walk to the lighted chamber within 45 seconds were recorded as "Pass" which means that the task was learned through reinforcement. The pass rate of five consecutive trials was then recorded as PC0 (0 hr postconditioning).

\section{Determination of short-term memory deficits}

Each fly was then returned inside its food vial and was left alone for six hours after training and PC0 recordings. After 6 hours, the fly was subjected to 5 more trials of the same test. The pass rate was recorded as PC6 (6 hours postconditioning) and served as an indicator of short-term memory.

\section{RESULTS AND DISCUSSION}

\section{Antioxidant activity of $G$. binucao crude leaf extract}

The computed EC50 value for the G. binucao crude leaf extract was $186.5 \mu \mathrm{g} / \mathrm{mL}$, and for the positive control, ascorbic acid, $4.037 \mu \mathrm{g} / \mathrm{mL}$. At $400 \mu \mathrm{g} / \mathrm{mL}$ sample concentration, the percent scavenging activity for the $G$. binucao crude leaf extract was $37.76 \%$, compared to $81.47 \%$ for ascorbic acid.

\section{G. binucao increased learning function of $D$. melanogaster}

In flies not exposed to ethanol, there was an $11.10 \%$ increase in the learning function in flies treated with high dose G. binucao compared to untreated flies (Figure 1). However, no significant effect was reported across all treatments after conducting one-way ANOVA and Dunn's multiple comparisons test. No significance was also noted for the individual sexes.

\section{G. binucao significantly increased short-term memory function on $D$. melanogaster}

In flies not exposed to alcohol, flies given a high dose of the extract displayed significantly increased memory function $(\mathrm{p}=0.0142)$, with a $33.97 \%$ increase in pass rates compared to untreated flies. Upon taking sex-specific data, females displayed a similar trend $(\mathrm{p}=0.0143)$ with a $45.86 \%$ increase in pass rate, while males did not. It should be noted that males treated with the high dose had a $16.94 \%$ increase in pass rates compared to the untreated flies (Figure 2).

\section{Effect of $G$. binucao on $D$. melanogaster mortor function with chronic alcohol exposure}

Chronic alcohol exposure significantly decreased the climbing pass rates of flies in the untreated group $(p=0.0040$, unpaired two-tailed t-test) (Figure 3). Climbing pass rates of flies upon chronic alcohol exposure did not significantly differ in flies treated with high dose $G$. binucao ( $\mathrm{p}>0.05$, unpaired two-tailed t-test). Interestingly, in flies treated with low dose G. binucao, chronic alcohol exposure significantly increased the climbing pass rate $(\mathrm{p}=0.0025$, unpaired two-tailed t-test $)$.

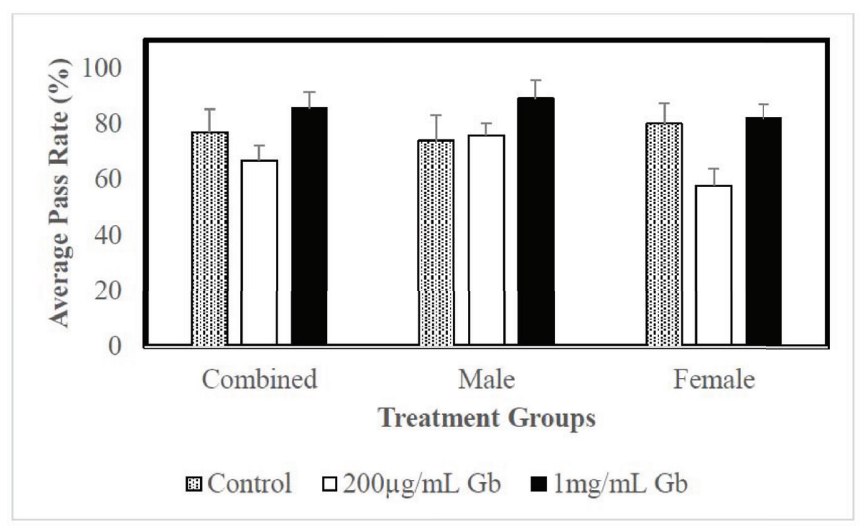

Fig. 1: Learning function of D. melanogaster after 20 days of treatment with a low dose and high dose G. binucao. Average pass rates of flies (combined sexes, male, female) treated with control (food only), $200 \mu \mathrm{g} / \mathrm{mL}$ G. binucao (low dose $\mathrm{Gb}$ ), and $1 \mathrm{mg} / \mathrm{mL} \mathrm{G}$. binucao (high dose $\mathrm{Gb}$ ) for 20 days, were determined using the Aversive Phototaxis Suppression Assay.

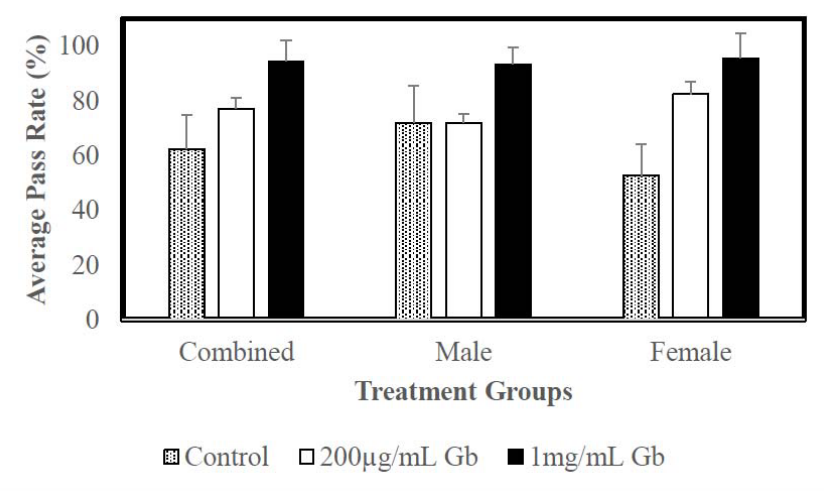

Fig. 2: Short-term memory function of D. melanogaster after 20 days of treatment with low dose and high dose G. binucao. Average pass rates of flies (combined sexes, male, female) treated with control (food only), $200 \mu \mathrm{g} / \mathrm{mL} G$. binucao (low dose $\mathrm{Gb}$ ), and $1 \mathrm{mg} / \mathrm{mL}$ G. binucao (high dose $\mathrm{Gb}$ ) for 20 days, were determined using the Aversive Phototaxis Suppression Assay six hours after training.

Tukey's multiple comparison tests of one-way ANOVA showed that climbing pass rate of flies chronically exposed to alcohol treated with low dose G. binucao were significantly higher than the high dose group $(\mathrm{p}=0.0333)$ but not with the untreated group $(p>0.05)$ (Figure 3A). Tukey's multiple comparison tests of one-way ANOVA showed no significant differences between all-male groups $(p>0.05)$ and all female groups $(p>0.05)$ (Figure 3B, 3C).

Male flies have higher geotactic activity after chronic alcohol exposure compared to female flies. This means that male flies are more resistant to sedation and show lesser impairment in motor function upon alcohol exposure. It was observed in a previous study that male flies are more resistant to ethanol sedation than female flies. Slower metabolism of ethanol was observed in 
females compared to males, leading to an increased concentration of ethanol in the body, which then results in greater sedation sensitivity in females (Devineni and Heberlein, 2013).

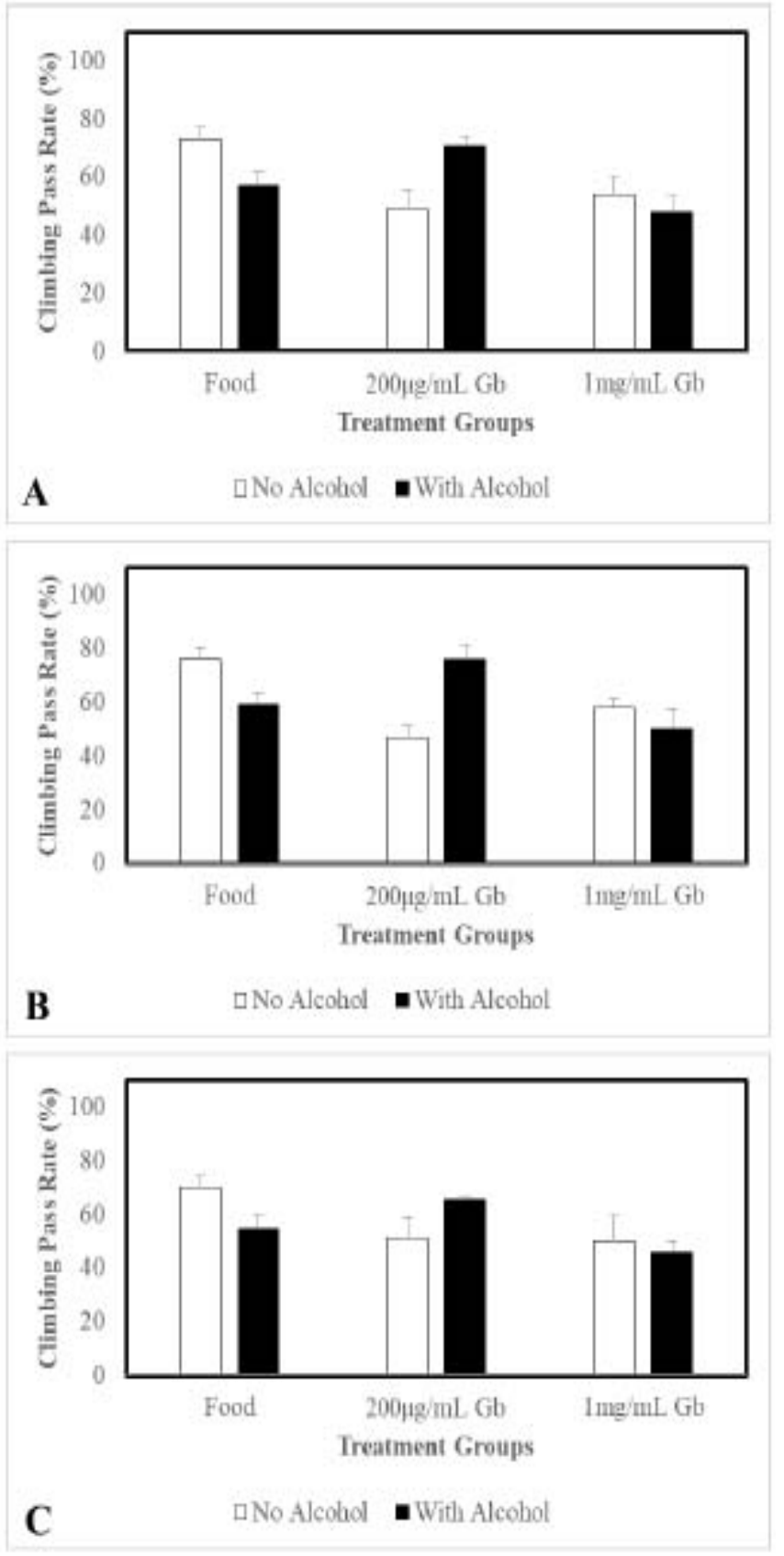

Fig. 3: Comparison of motor function of fruit flies using the negative geotaxis assay. (A) males and females combined, (B) males only, and (C) females only after 15 days of treatment with a low dose and high dose $\mathrm{Gb}$, in the presence and absence of chronic ethanol exposure $(n=30$ per replicate).

It was previously studied that chronic alcohol exposure causes loss of postural control and negative geotactic activity in adult flies. It also results in motor impairment and sedation which can be attributed to the depressant nature of alcohol (Robinson et al., 2012). Chronic alcohol exposure can upregulate the sensitivity of NMDA receptors in the brain. This can result in a glutamateinduced cytotoxic response in the brain. There will be a calcium influx through the NMDA receptors, which will then be taken up in the mitochondria causing the generation of reactive oxygen species that can disrupt the function of the mitochondria and plasma membranes. This results in impairments in neurological functions such as abstract problem solving, visuospatial and verbal learning, memory function, perceptual motor skills and even motor function (Harper and Matsumoto, 2005).

\section{G. binucao prevents learning deficits from chronic alcohol exposure of $D$. melanogaster}

In untreated flies, a significant $37.23 \%$ decrease in learning function ( $\mathrm{p}=0.0251$ ) was noted (Figure 4 ) upon chronic alcohol exposure. Treatment with high dose and low dose $G$. binucao prevented this reduction in learning function. The learning function of flies treated with high dose G. binucao was $43.69 \%$ higher $(\mathrm{p}<0.0001)$ compared to the untreated group (Figure 4A). Upon analysis of the sex-specific data set, females (Figure 4C) maintained a significant increase $(p=0.0009)$ while males (Figure $4 B)$ did not exhibit a significant increase $(p=0.1200)$ in learning function.

\section{G. binucao prevents short-term memory deficits from chronic alcohol exposure of $D$. melanogaster}

There was an observed 33\% reduction (see Figure 5A) in the short-term function of the flies after chronic alcohol exposure. However, no significance was observed upon doing statistical analysis. In flies chronically exposed to ethanol, treatment with a high dose of the extract significantly improved short-term memory compared to the untreated group $(\mathrm{p}<0.0001)$. For the male-only set-ups (Figure 5B), significant differences were seen between the untreated and low dose groups $(\mathrm{p}=0.0309)$, and the untreated and high dose groups $(\mathrm{p}<0.0001)$, with the most significant change seen between the untreated and high dose groups. For the female set-ups (Figure 5C), a significant increase was noted between the low-dose and high-dose set-ups $(\mathrm{p}=0.0159)$. Between the food and high-dose set-up, a p-value of 0.0556 was recorded indicating an almost significant difference.

There was an increase in the short-term memory functionin untreated female flies upon chronic alcohol exposure but no statistical significance was observed. However, the shortterm memory function in untreated male flies significantly decreased by $86 \%$ with chronic alcohol exposure. There was no significant increase reported between the ethanol-exposed and non-ethanol exposed set-ups with low and high dose extract treatments. There was no significant difference in the DMSO setups across all experiments.

There was also an observed sexual dimorphism in cognitive ability: learning deficit was more profound in female flies chronically exposed to alcohol while short-term memory function was more impaired in male flies. These findings may be attributed to differences in neuronal and metabolic activities between male and female flies. Based on a previous study, there is little evidence for sex-specific neuronal regulatory adaptation in the brain, so differences observed may be more due to metabolic tolerance to alcohol rather than inherent cognitive abilities of the flies (Catalan et al., 2012).

Female flies are generally larger in size compared to males, which may have contributed in the differences in the total amount of intake (Testa et al., 2013; Wong et al., 2009). In 
acute alcohol exposure, Paul Mark B. Medina, Biological Models Laboratory, Department of it has been shown that male flies have increased ethanol hyperactivity and a greater resistance to ethanol sedation compared to female flies (Devineni and Heberlein, 2013). These differences in hyperactivity and resistance in acute settings may also explain the difference in chronic settings, where tolerance could have affected the overall toxic effect of alcohol. The observed greater impairment in short-term memory function in males, however, is not explained by this finding and may be due to a different mechanism.
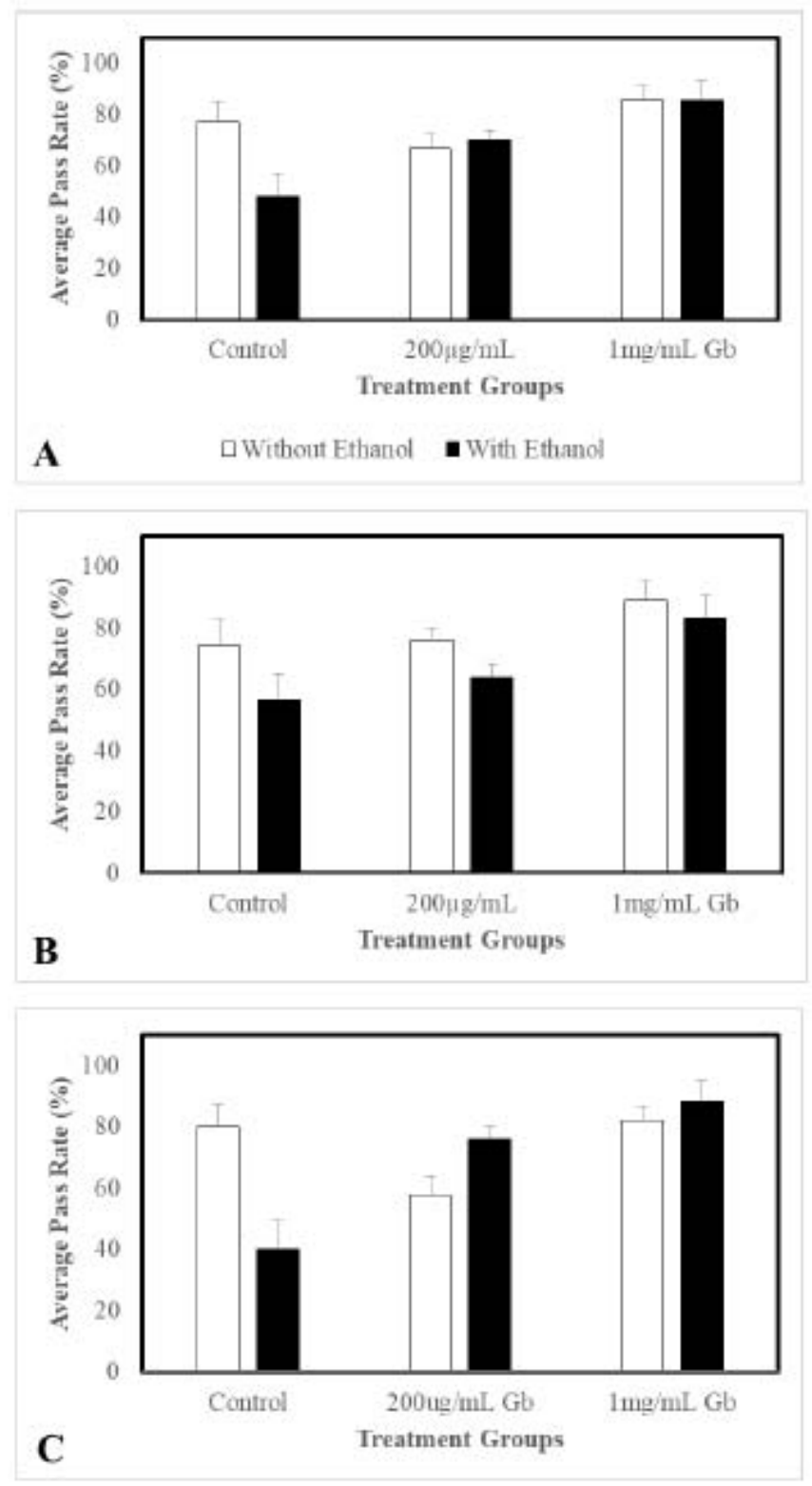

Fig. 4: Learning functions of fruit flies using the aversive phototaxis suppression assay. (A) males and females average, (B) males only, and (C) females only, in the presence and absence of chronic ethanol exposure ( $n=30$ per replicate).

\section{Phytochemical Analysis of G. binucao Crude Leaf Extract}

Upon phytochemical analysis, G. binucao crude leaf extract tested positive for carbohydrates and reducing sugars, proteins and amino acids, triterpenes, quinones, and flavonoids (Table 1).

Phytochemical analysis of the G. binucao fruit has been done before but there have been no studies on its leaves. Xanthones ( $\alpha$-mangostin, gartanin, and isomangostin), sterols ( $\beta$-sitosterol and stigmasterol), and monosaturated and saturated triglycerides have been isolated from the pericarp of $G$. binucao (Ragasa et al., 2014).

Table 1: Phytochemicals present in G. binucao.

\begin{tabular}{lc}
\multicolumn{1}{c}{ Phytochemicals } & Indication \\
\hline Carbohydrates and Reducing Sugars & + \\
Proteins and Amino Acid & + \\
Alkaloids & - \\
Glycosides & - \\
Steroids and Phytosterols & + \\
Terpenes and Terpenoids & + \\
Quinones & - \\
Anthraquinones & - \\
Saponins & + \\
Flavonoids & - \\
Phenols & - \\
Tannins &
\end{tabular}

$(+)$ - present; (-) - absent.

Phytochemical analysis of various extracts from other Garcinia species have been done in several studies before, and among the compounds isolated were hydroxycitric acid, flavonoids, terpenes, polysaccharides, procyanidines and polyisoprenylated benzophenone and xanthone derivatives. Xanthones have been found in most species and parts of the plant. In leaves, xanthones, quinones, garcinol, stigmasterol, flavones, among others, have been reported to be present (Hemshekhar et al., 2011). More than 60 xanthones have also been isolated from different parts of Garcinia mangostana, as well as benzophenones, flavonoids, and anthocyanins. In G. mangostana leaves, 1,5,8-Trihydroxy-3methoxy-2-(3-methylbut-2-enyl) xanthone and 1,6-Dihydroxy-3methoxy-2-(3-methyl-2-buthenyl)-xanthone have been identified (Obolskiy et al., 2009).

These phytochemicals may be involved in the neuroprotective properties of GBE. Flavonoids can induce brain perfusion, stimulate angiogenesis, neurogenesis, and changes in the morphology of neurons involved in learning and memory. They have also been shown to inhibit neuronal death caused by neurotoxicants and promote synaptic plasticity (Nehlig, 2012). Several xanthones from $G$. mangostana have reported hydroxyl radical scavenging activity in different assays, including the DPPH assay (Obolskiy et al., 2009). An isolated bioflavanoid complex of Garcinia kola seed, Kolaviron, was also shown to inhibit ethanol-induced oxidative stress in the liver of experimental rats (Hemshekhar et al., 2011). Further phytochemical analysis, screening for various other compounds, especially xanthones, and identifying more specific compounds is highly recommended to identify the active components of the extract. 

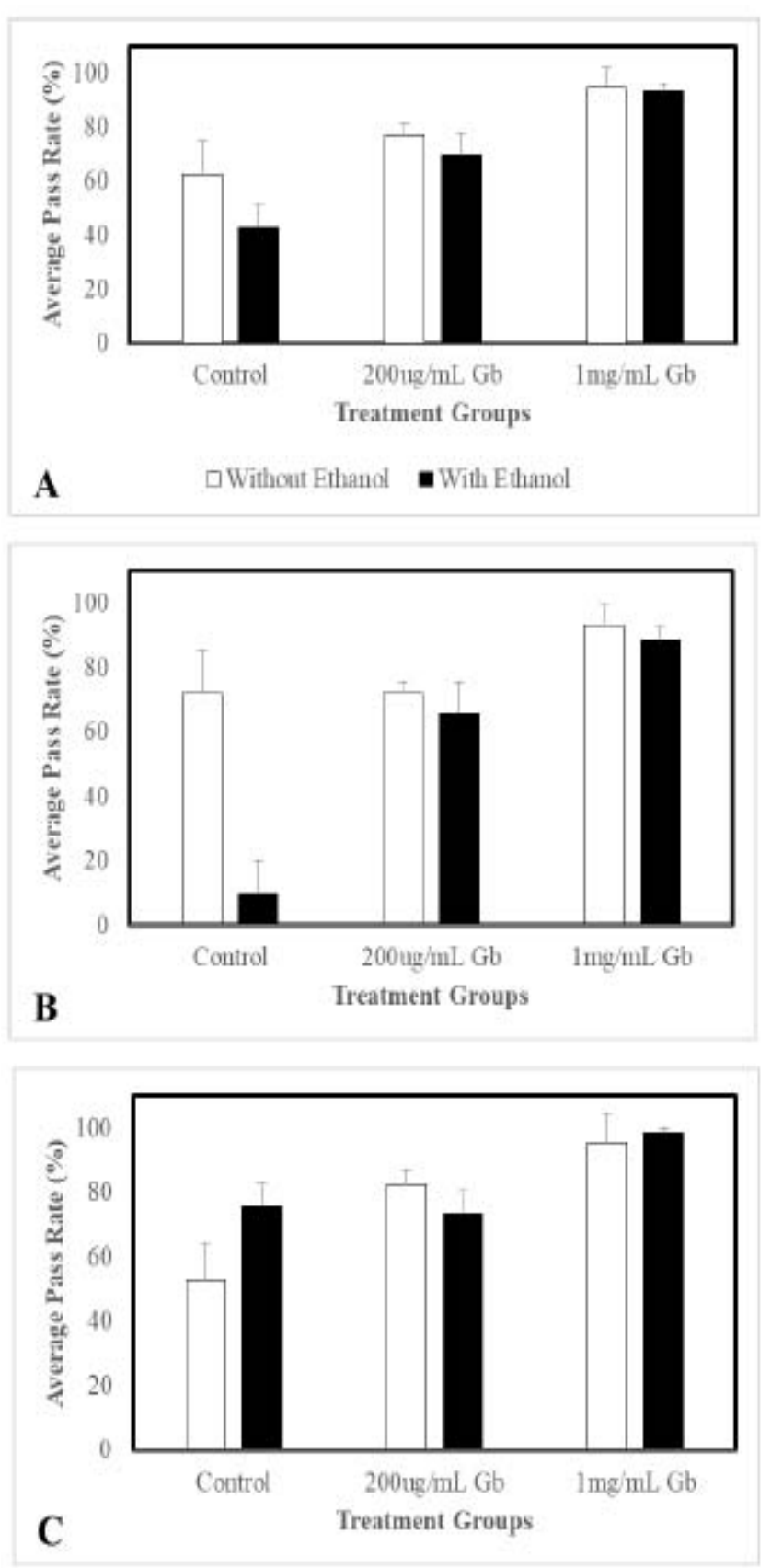

Fig. 5: Short-term memory functions of fruit flies using the aversive phototaxis suppression assay. (A) males and females average, (B) males only, and (C) females only with and without chronic ethanol exposure $(n=30$ per replicate).

Although the antioxidant level of GBE is low, it still can contribute minimally to the neuroprotective properties of GBE. However, the neuroprotection that we have observed may be largely attributed to another mechanism that is not dependent on the direct radical or oxidant scavenging properties of the phytochemicals in GBE. Previous literature have shown that flavonoids can prevent the oxidant-induced neuronal injury by modulating a number of protein kinase and lipid kinase signaling cascades such as the PI3 kinase (PI3K)/Akt, tyrosine kinase, protein kinase C (PKC) and mitogen-activated protein kinase (MAP kinase) signalling pathways (Schroeter et al., 2001). Flavonoids have also been shown to prevent the activation of caspase- 3 which prevent neuronal apoptosis and activation/phosphorylation of signalling proteins important in the pro-survival pathways (Vauzour et al., 2007).

\section{CONCLUSION}

G. binucao crude ethanolic leaf extract supplementation prevented motor dysfunction and cognitive deficits due to chronic alcohol exposure, and enhanced motor, learning and short-term memory functions in $D$. melanogaster. Preliminary testing also showed that the extract exhibited minimal antioxidant activity despite the presence of phytochemicals such as triterpenes, quinones, and flavonoids. Other mechanisms aside from the antioxidant activity may be responsible for the protective properties of $G$. binucao against alcohol-induced neurotoxic effects on motor, learning, and short-term memory functions.

\section{ACKNOWLEDGMENTS}

This research was funded by the UP College of Medicine and UPM NIH Student Researcher Grant 2016. We would also like to acknowledge Dr. Raymond Oliva for his inputs in the proposal, and the UP College of Medicine Therapeutics 202 Group 7 AY 2016-2017 (Frinz Rubio, Yasmin Salces, JM Santiago, Charity Santos, Luis Santos, Noel Sarrosa, Kevin Sese, Clyde Silverio, Ana Sison, Jamie Sta. Maria, JR Sta. Maria, Karol Tablante, Olin Tamayo, Bea Tan, Randell Tan, Riem Teves, Joyce Tiam-Lee) for their invaluable assistance in the conduct of all laboratory experiments and support in the completion of this project.

\section{CONFLICTS OF INTEREST}

The authors declare no conflict of interest. The funding sponsors had no role in the design of the study; in the collection, analyses, or interpretation of data; in the writing of the manuscript, and in the decision to publish the results.

\section{REFERENCES}

Berger KH, Kong EC, Dubnau J, Tully T, Moore MS. Ethanol Sensitivity and Tolerance in Long-Term Memory Mutants of Drosophila melanogaster. Alcohol Clin Exp Res, 2011; 32(5):895-908.

Catalán A, Hutter S, Parsch J. Population and sex differences in Drosophila melanogaster brain gene expression. BMC Genomics, 2012; 13:654.

Chua-Barcelo RT. Ethno-botanical survey of edible wild fruits in Benguet, Cordillera administrative region, the Philippines. Asian Pac J Trop Biomed, 2014; 4:S525-38.

Devineni AV, Heberlein U. The Evolution of Drosophila melanogaster as a Model for Alcohol Research. Annu Rev Neurosci, 2013; $36: 21-128$

Dong Y, Guha S, Sun X, Cao M, Wang X, Zou S. Nutraceutical Interventions for Promoting Healthy Aging in Invertebrate Models. Oxid Med Cell Longev, 2012; 718491.

French RL, Heberlein U. Glycogen synthase kinase-3/Shaggy mediates ethanol-induced excitotoxic cell death of Drosophila olfactory neurons. Proc Natl Acad Sci USA, 2009; 106(49):20924-20929

Gutierrez-Orozco, Failla ML. Biological Activities and Bioavailability of Mangosteen Xanthones: A Critical Review of the Current Evidence. Nutrients, 2013; 5(8):3163-3183..

Harper C, Matsumoto I. Ethanol and brain damage. Curr Opin Pharmacol, 2005; 5(1):73-78.

Hemshekhar M, Sunitha K, Sebastin Santhosh M, Devaraja S, Kemparaju K, Vishwanath BS, Niranjana SR, Girish KS. An overview on 
genus garcinia: phytochemical and therapeutical aspects. Phytochem Rev, 2011; 10:325-351.

Maples T, Rothenfluh A. A simple way to measure ethanol sensitivity in flies. J Vis Exp, 2011; 48:e2541.

Nehlig A. The neuroprotective effects of cocoa flavanol and its influence on cognitive performance. Br J Clin Pharmacol, 2013; 75(3):71627

Obolskiy D, Pischel I, Siriwatanametanon N, Heinrich M. Garcinia mangostana L.: A Phytochemical and Pharmacological Review. Phytother. Res., 2009; 23:1047-1065.

Oscar-Berman M, Marinkovi K. Alcohol: Effects on Neurobehavioral Functions and the Brain Marlene, Neuropsychol Rev, 2014; 17:239-257.

Pedraza-Chaverrí J, Reyes-Fermín LM, Nolasco-Amaya EG, Orozco-Ibarra M, Medina-Campos ON, González-Cuahutencos O, RiveroCruz I, Mata R. ROS scavenging capacity and neuroprotective effect of a-mangostin against 3-nitropropionic acid in cerebellar granule neurons, Exp Toxicol Pathol, 2009: 61:491-501.

Prieto P, Pineda M, Aguilar M. Spectrophotometric Quantitation of Antioxidant Capacity through the Formation of a Phosphomolybdenum Complex: Specific Application to the Determination of Vitamin E. Anal Biochem, 1999; 269:337-341.

Quevedo ES, Pham LJ, Merca FE, Laurena AC. Physicochemical Properties, Lipid and Fatty Acid Profile of "Batuan" [Garcinia binucao (Blco.) Choisy] Seed Oil. Ann Trop Res, 2015; 37(2):11-22.

Ragasa C, Torres O, Marasigan E, Shen C. Sterols and triglyceride from the fruit of Garcinia binucao. Der Pharma Chemica, 2014, 6(6):229-232.

Robinson BG, Khurana S, Pohl JB, Li W, Ghezzi A, Cady AM, Najjar K, Hatch MM, Shah RR, Bhat A, Hariri O, Haroun KB, Young MC, Fife, Hooten J, Tran T, Goan D, Desai F, Husain F, Godinez RM, Sun JC, Corpuz J, Moran J, Zhong AC, Chen WY, Atkinson NS. A Low Concentration of Ethanol Impairs Learning but Not Motor and Sensory Behavior in Drosophila Larvae. PLoS ONE, 2012; 7(5):e37394.

Rukachaisirikul V1, Adair A, Dampawan P, Taylor WC, Turner PC. Lanostanes and friedolanostanes from the pericarp of Garcinia hombroniana. Phytochemistry, 2000; 55(2):183-8.
Schroeter H, Spencer JP, Rice-Evans C, Williams RJ. Flavonoids protect neurons from oxidized low-density-lipoprotein-induced apoptosis involving c-Jun N-terminal kinase (JNK), c-Jun and caspase-3. Biochem J, 2001; 30:1211-1219

Tangpong J, Miriyala S, Noel T, Sinthupibulyakot C, Jungsuwadee P, Clair DKS. Doxorubicin-induced Central Nervous System Toxicity and Protection by Xanthone Derivative of Garcinia mangostana. Neuroscience, 2012; 175:292-299.

Testa ND, Ghosh SM, Shingleton AW. Sex-Specific Weight Loss Mediates Sexual Size Dimorphism in Drosophila melanogaster. PLoS One, 2013; 8(3):e58936

Vauzour D, Vafeiadou K, Rice-Evans C, Williams RJ, Spencer JP. Activation of pro-survival Akt and ERK1/2 signalling pathways underlie the anti-apoptotic effects of flavanones in cortical neurons. J Neurochem, 2007; 103(4):1355-67.

Velasco AJD, Medina PMB. Crude Anthocyanin Extract (CAE) from Ballatinao Black Rice Does Not Alter Longevity and Increases Stress Susceptibility of Drosophila melanogaster. Int J Curr Res Biosci Plant Biol, 2014; 1(4):35-42.

Vinluan M. Adolescent and Youth Health Program (AYHP), Dep. Heal. (2003). [ONLINE] Available at http://www.doh.gov.ph/adolescentand-youth-health-program [Accessed 26 December 2017]. 2003.

Wong R, Piper MDW, Wertheim B, Partridge L. Quantification of Food Intake in Drosophila. PLoS One, 2009; 4(6):e6063.

World Health Organization, Global Status Report on Alcohol and Health 2014, WHO Press, World Health Organization, 20 Avenue Appia, 1211 Geneva 27, Switzerland, 2014.

How to cite this article:

Tantengco OAG, Tan JJE, Tan NRC, Sison MCC, Medina PMB. Garcinia binucao crude ethanolic leaf extract prevents alcoholinduced neurotoxic effects on learning, short-term memory, and motor functions in Drosophila melanogaster. J App Pharm Sci, 2018; 8(10): 106-112. 